\title{
diffusion-înolarrentals
}

The Open-Access Journal for the Basic Principles of Diffusion Theory, Experiment and Application

\section{New Options for Measuring Molecular Diffusion in Zeolites by MAS PFG NMR}

\author{
Moisés Fernandez, André Pampel, Dieter Freude, Jörg Kärger
}

Universität Leipzig, Fakultät für Physik und Geowissenschaften, Linnéstraße 5, 04103

Leipzig, Germany, E-Mail: moises@physik.uni-leipzig.de

\section{Introduction}

Combination of pulsed field gradient (PFG) NMR with magic-angle spinning (MAS) NMR is demonstrated to have remarkable advantages in comparison with conventional PFG NMR, if applied to diffusion measurements in beds of nanoporous particles, notably zeolites. These advantages are based on the effect of line narrowing and concern (i) a prolongation of the intervals during which the magnetic field gradients may be applied and a corresponding enhancement in the sensitivity towards small molecular displacements, and (ii) an enhanced resolution in the chemical shift scale. Both options are illustrated by measuring the diffusion coefficient of n-butane adsorbed in silicalite-1. ${ }^{[1]}$

\section{Studying Diffusion of Molecules sorbed in Zeolites using MAS PFG NMR}

NMR diffusion measurements are based on recording the differences in the precessional phases of any individual spin of the nuclei under study, accumulated during the application of the first and second gradient pulse trains, i.e. during the time interval during which an additional, inhomogeneous field is superimposed upon the constant magnetic field. Because the amplitude of the magnetic field gradient pulses is limited, sensitivity enhancement towards smaller displacements has to be based on an enhancement of the total time of gradient application. Long magnetic field gradient pulses, in turn, imply correspondingly long periods of time within the PFG NMR pulse sequences, during which nuclear magnetization is oriented in the plane perpendicular to the direction of the magnetic field. The application of magnetic field gradient pulses of long duration is limited, therefore, by the rate of the decay of transverse magnetization. ${ }^{[2}$,

In heterogeneous systems, there are essentially two major mechanisms giving rise to a decay of the transverse proton magnetization, viz. (i) a sustainable dipolar interaction between the spins under consideration and (ii) differences in the internal magnetic fields, brought about by sample heterogeneities and the consequent heterogeneities in the susceptibility. Those disturbing influences, which will also result in reduced spectral resolution, can be drastically reduced by magic-angle spinning (MAS).

The contribution describes investigations that mainly profit from the elimination of the disturbing influence of the inhomogeneity of the sample susceptibility playing a decisive role for PFG NMR diffusion measurements of molecules in beds of zeolites. ${ }^{[4,5]}$

In general, NMR spectra of polycrystalline materials are broadened by anisotropic interactions such as dipolar coupling, chemical shift anisotropy, quadrupolar coupling, 
and susceptibility effects. Depending on their strength and the achievable rotation frequency, MAS can reduce or even completely suppress these effects during the time course of an NMR experiment. If, in addition to the motion of the spins by the sample rotation, thermal motions are stochastically modulating the NMR interactions, both effects will compete in their influence on the time evolution of the spin system.

In order to evaluate the susceptibility effects on the spectra of our systems, we have measured additional MAS NMR spectra of ethane sorbed in silicalite-1 at different rotation frequency. The broadness of the spectra without MAS and thus, the fast decay of the transverse magnetization could be explained by a theoretical model taking into account MAS and thermal motion. ${ }^{[5,6]}$

Using a $750 \mathrm{MHz}$ spectrometer, at a rotation frequency of $10 \mathrm{kHz}$, the spectrum of $\mathrm{n}$ butane is observed with a residual line width of $44 \mathrm{~Hz}$ and $73 \mathrm{~Hz}$ of the signals of the $\mathrm{CH}_{3^{-}}$and $\mathrm{CH}_{2}$-groups, respectively. The performance of the PFG NMR diffusion experiment is straightforward under these conditions. The diffusion coefficient calculated from the decay of integrals of the signal of the $\mathrm{CH}_{3}$ peak is $3.17 \cdot 10^{-10} \mathrm{~m}^{2} \mathrm{~s}^{-1}$.

\section{Conclusions and Outlook}

For the first time, PFG NMR and MAS NMR have been combined to measure intracrystalline zeolitic diffusion. It has been illustrated by model calculation and demonstrated experimentally that in this way line broadening due to the susceptibility heterogeneities inevitable in such systems may be dramatically reduced. Thus, one of the most decisive limitations of the application of PFG NMR to zeolitic host-guest systems, viz. the heterogeneity-induced line broadening and the associated rolling back of the total time over which the magnetic field gradient may be applied, may be overcome.

Higher gradient strength will enable recording of much slower diffusion processes over shorter observation times, facilitating in this way the exploration of intracrystalline diffusion phenomena.

\section{References}

[1] A. Pampel, M. Fernandez, D. Freude, J. Kärger, Chem. Phys. Lett. 2005, 407, 53.

[2] J. Kärger, in Encyclopedia of Nuclear Magnetic Resonance, Vol. 3 (Eds.: R. K. Harris, M. Grant), Wiley, Chicester, 1996, pp. 1656.

[3] P. T. Callaghan, Principles of Nuclear Magnetic Resonance Microscopy, Clarendon Press, Oxford, 1993.

[4] D. Michel, A. Pampel, J. Roland, J. Chem. Phys, 2003, 119, 9242.

[5] J. Roland, D. Michel, Magn. Res. Chem. 2000, 38, 587.

[6] D. Fenzke, B. C. Gerstein, H. Pfeifer, J. Magn. Reson. 1992, 98, 469. 